doi:10.1016/j.vaccine.2006.03.022

Copyright (c) 2006 Elsevier Ltd All rights reserved.

\title{
Developing a vaccination evaluation model to support evidence-based decision making on national immunization programs
}

\author{
Tjeerd G. Kimman ${ }^{a}$ *, Hein J. Boot ${ }^{a}$, Guy A.M. Berbers ${ }^{a}$, Patricia E. \\ Vermeer-de Bondt ${ }^{b}$, G. Ardine de Wit $^{c}$ and Hester E. de Melker
}

* Corresponding author. Tel.: +3130274 2330; fax: +3130274 4449.

a Laboratory for Vaccine-Preventable Diseases, National Institute of Public Health and the Environment, P.O. Box 1, 3720 BA Bilthoven, The Netherlands ${ }^{\mathrm{b}}$ Centre for Infectious Diseases Epidemiology, National Institute of Public Health and the Environment, P.O. Box 1, 3720 BA Bilthoven, The Netherlands ${ }^{\circ}$ Department of Prevention and Health Services Research, National Institute of Public Health and the Environment, P.O. Box 1, 3720 BA Bilthoven, The Netherlands

Received 18 November 2005; revised 3 March 2006; accepted 7 March 2006. Available online 27 March 2006.

\begin{abstract}
Among all public health provisions national immunization programs (NIPs) are beyond doubt one of the most effective in reducing mortality, morbidity, and costs associated with major infectious diseases. To maintain their success, NIPs have to modernize in response to many new and old demands regarding efficacy, safety, availability of new vaccines, emerging and evolving pathogens, waning immunity, altered epidemiological situations, and the public's trust in the program. In this paper we present an evaluation model in the form of a checklist that may help in collecting relevant scientific information that is necessary for evaluation and decision making when considering changes in a NIP. Such a checklist points to relevant information on the vaccine-preventable disease, the pathogen causing it, the vaccine, and the cost-effectiveness ratio of the vaccine. However, the final judgment on a potential change in the NIP cannot be based on a simple algorithm, as the relevant information reflects factors of a very different kind and magnitude, to which different value judgements may be added, and which may have certain degrees of uncertainty. Because any change in the NIP may be accompanied by more or less unforeseen changes in the vaccine's efficacy, evolutionary consequences, including the antigenic composition of the pathogen, and the vaccine's safety profile, an intensive surveillance program should accompany any NIP. Elements thereof include clinical-epidemiological surveillance, surveillance of vaccination coverage, immune surveillance, surveillance of microbial population dynamics, and surveillance of adverse events and safety issues. We emphasize that the decision to introduce a vaccine in the NIP should be taken as seriously, both scientifically and ethically, as the decision to withhold a vaccine from the NIP. In the latter case one might be responsible for vaccine-preventable disease and mortality.
\end{abstract}


Keywords: National immunization program; Vaccine-preventable disease; Vaccine; Pathogen; Disease; Cost-effectiveness; Vaccination

\section{Introduction}

Most countries offer programmatic vaccination to their inhabitants, in particular to their infants and young children. These national immunization programs (NIPs) are beyond doubt one of the most effective disease prevention strategies available today, both in reducing mortality and morbidity, and in being costsaving or cost-effective. These NIPs can still be very much improved in many developing and developed countries, esp. their coverage and the speed of introduction of new vaccines. In addition, effective vaccines against major killers as malaria and AIDS are still not available. These NIPs especially developed during the 1950s when effective vaccines against polio, pertussis, diphtheria, and tetanus had become available. Although the NIPs of many, especially developed, countries are quite similar, a recent review also showed substantial differences in vaccination schedules, responsible authorities, organization structures (the difference being more or less centralized), government control, ways of financing, political support, and, most importantly, levels of compliance [1]. In many countries these programs evolved gradually in response to the epidemiological situation, and the availability of vaccines and financial resources. Their long and successful history, however, is no reason to restrain from modernization. We therefore want to stimulate a pro-active policy regarding their further development. The emergence or disappearance of infections, new insights in epidemiological or immunological parameters, and the development of better vaccines continuously urge changes in the vaccination program. Among the challenges are: (i) the development of "difficult" vaccines, such as those directed against human immunodeficiency virus (HIV), respiratory syncytial virus (RSV), Plasmodium falciparum, and Neisseria meningitidis serogroup B; (ii) the maintaining of a high vaccination coverage and the public's confidence in the NIP; (iii) the programmatic implementation of newly developed vaccines; (iv) the adjustment of the vaccination schedule in response to evolving pathogens, new epidemiological situations, and changes in the population's immunity (for example, due to waning immunity and reduced exposure to circulating pathogens).

For example, poliovirus infection and vaccination may induce long-term immunological memory. However, a recent study showed that memory immunity in seronegative elderly did not protect against virus excretion, therewith posing the question whether elderly should be re-vaccinated against polio during an outbreak [2]. NIPs may require adaptations when vaccines come into the market that are now in the industry's pipeline, including new vaccines against well-known pathogens as influenza, RSV, rotavirus, meningococci, group A streptococci, against gastric infection caused by Helicobacter pylori, against sexually transmitted diseases caused by HIV and human papilloma virus (HPV), against tropical diseases as malaria and dengue fever, against emerging diseases as SARS and West Nile virus, and against perceived bioterrorist threats such as anthrax, small pox [3]. Furthermore, while many NIPs are directed to the youngest individuals, there is now a trend to protect adolescents, adults, and elderly against diseases to which they are more vulnerable because of their life stage or life style. Vaccinating elderly against varicella-zoster virus might, for example, protect against herpes zoster [4]. 
Ideally, all changes in NIPs should be based on evidence corroborating that such changes indeed improve public health in a (cost-)effective manner. Often the decision to vaccinate a population in the framework of a NIP is based on the same arguments as the decision to vaccinate an individual. However, program-specific or public health-relevant considerations also might lead to a decision that would differ if one would look at an individual's interests only. Here we present an evaluation model in the form of a checklist that may support decision making on a NIP. The checklist of questions may help to collect relevant scientific information that is necessary for evaluation and decision making. However, the final decision making depends on the evaluation of data of a very different kind and magnitude, as well as value judgments, which will be covered in Section 4. Interestingly (and illustrating the need for a structured analysis of vaccination programs), we noticed that during our work a Canadian group had adopted a somewhat similar approach [5]. Despite similarities in approach, some differences between their and our work illustrate that geographical, demographic, cultural, and political factors affect decision making on vaccination policy. To assess the usefulness of this approach, we have evaluated vaccination against varicellazoster virus in an accompanying paper [6]. Vaccination against varicella has become feasible, because a four-fold combination vaccine in which the measlesmumps-rubella (MMR) components are supplemented by a varicella component (MMRV) has recently been developed. In this paper we focus on the background of issues related to the introduction of new vaccines in NIPs.

\section{Methods}

The checklist has been assembled after discussions by a multidisciplinary team consisting of microbiologists, immunologists, epidemiologists, experts on vaccine safety, and health economists. The relevant information was assigned to one of the following fields: "vaccine", "disease", and "pathogen", which together gives input for the "cost-effectiveness analysis" (Fig. 1). All fields together contain the information allowing comprehensive considerations regarding the NIP. Lack of data on specific subjects points to uncertainties in decision making and research questions. 
VACCINE

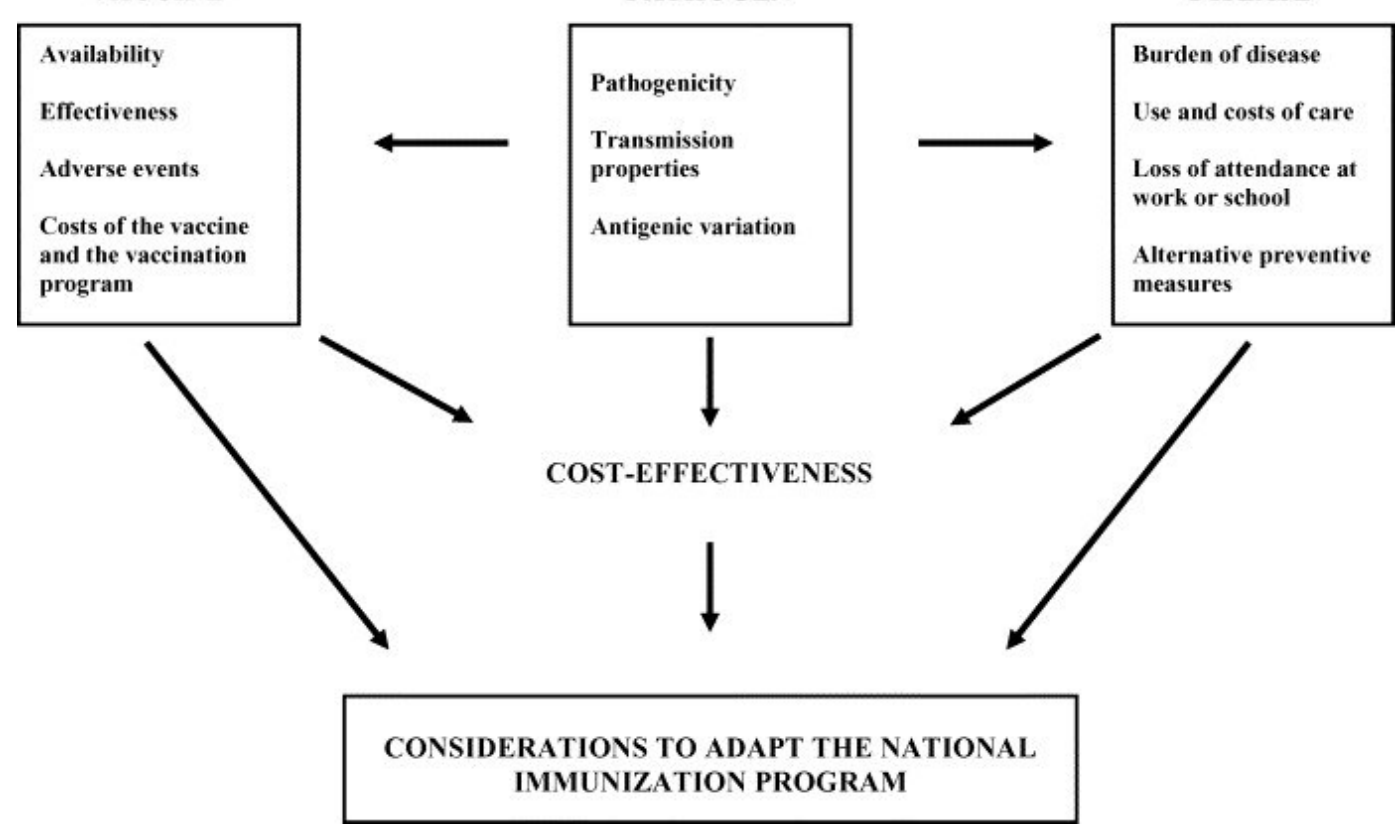

Fig. 1. Considerations to adapt the national immunization program.

\section{Results}

\subsection{An evaluation model to support decision making on NIPs}

The first and major goal of a NIP is to prevent disease and mortality, especially from diseases with a high burden of disease or that have a high mortality rate (for example, RSV and bacterial meningitis). The program should be acceptable and effective. Hence, it should lead to a minimum of discomfort and a maximum of protection as far as this is considered (economically) justifiable. Designing or improving a NIP is not easy. Information from different fields and sources is needed and should be collectively evaluated. A final decision is thus based on factors of very different kind and magnitude, and often with degrees of uncertainty. For example, both hard data on antigenic composition and price of a vaccine, and psychological factors, such as the public's anxiety on a presumed microbial threat, may all be necessary in the final judgment on the incorporation of a particular vaccine. Not only information on different subjects is needed (e.g., on available vaccines, microbial threats, burden of disease, cost-effectiveness, etc.), but such information is not stable in time and sometimes unknown, and therefore needs constant updating and evaluation. We do favor that emotional factors are counterbalanced by scientific data, although this may face limits. Hence, a final decision cannot be based on a simple algorithm, and is finally a political choice. Value judgments, sometimes only implicit, are therefore important when weighing scientific data as well. Science cannot do more than presenting the most reliable data to help making such a decision. The different elements of all assembled information may be given different weight in the final decision. Other relevant factors (political pressure, public anxiety, and lack of financial resources) may be given a high, or even higher, priority in comparison with the more objectively assembled scientific and economic data. The outcome of the cost-effectiveness analysis, together with characteristics of the vaccine, the pathogen, and the disease, is input for the final judgment on a proposed change of the NIP. 
As outlined in Table 1, we decided that the checklist should contain information regarding the vaccine on:

- Availability of vaccine, potential combination vaccines, and suitability to be incorporated in the NIP.

- Effectiveness of the vaccine on the short- and long-term, taking factors as vaccine characteristics, disease, composition of the NIP, structure of the population, and molecular evolution of the pathogen into account.

- The vaccine's safety profile, reflecting knowledge of nature and frequency of short- and long-term adverse events.

- Costs of the vaccine and the vaccination program, both once-only costs for introduction of the vaccine, and annual costs.

- Chances of successful implementation of the vaccine. This is apparently a complex issue. Many studies have investigated the reasons for poor compliance and have provided varying conclusions. The reasons for poor compliance (in various settings) include lack of an effective primary care system, complexity of the vaccination schedule, the costs of the vaccines, problems with access tot health care services, the need for multiple injections at a single visit, lack of parental awareness, competing parental priorities, parental complacency, long waiting times in public clinics, lack of reliable transportation, lack of reminders, inappropriate interpretation of contraindications, poor records keeping, and concerns regarding adverse reactions to vaccines [7] and [8]. All these aspect may require special attention or specific measures, for example, introduction of combination products, safer products, or education.

Table 2 details relevant information of the pathogen, and includes information on:

- Pathogenicity, risk groups, interaction with other pathogens, potential consequences of routine vaccination, for example, caused by a vacant felling ecological niche that may result in the emergence of other pathogens.

- Transmission properties, in order to estimate the effect of vaccination on the pathogen's transmission characteristics in the population and subpopulations thereof. Such information may be relevant in deciding whether vaccination should be universal or targeted at specific groups, and is thus relevant in the costeffectiveness analysis.

- Antigenic variation, which may diminish the vaccine's efficacy in the short- or long-term.

Relevant knowledge of the target disease (Table 3) comprises information on:

- Burden of disease in the whole population, and in specific age and risk groups.

- The impact of disease on society, i.e. the way and degree the disease may disturb social structures.

- Use of medical services and associated costs of the disease. 
- Absenteeism from school and work. Although such knowledge may not be decisive, it gives insight into societal aspects of the disease and potential benefits of vaccination thereupon.

- Alternative preventive measures, giving insight into the effectiveness and costs of such measures in comparison with vaccination.

The cost-effectiveness analysis (Table 4) gives information on:

- The cost-utility ratio for society. Alternative analyses include a costeffectiveness ratio per case prevented, a cost-consequence ratio, or a costbenefit ratio.

- Indirect effectiveness that is afforded when family members of vaccinated children experience less illness, as described for example, for influenza vaccination [9].

- Direct costs of immunization (vaccine, syringes, personnel, administrative expenses).

- Indirect costs (time of parents and caregivers, cost savings for parents who do not need take time off from work to care for a sick child).

- Whether the vaccine is cost-saving, cost-effective, or not. A vaccine is usually considered cost-effective in The Netherlands when a QALY is gained for less than $20,000 €$, intermediate when a QALY is gained for $20,000-50,000 €$, and not cost-effective when a QALY is gained for $50,000 €$ or more.

\section{Discussion}

\subsection{The role of cost-effectiveness analyses}

A cost-effectiveness analysis may take a central role in the decision-making process, emphasizing the crucial role of objective and quantitative information, and the need to spend money on health fairly and effectively. Nonetheless, considerations that emphasize specific or qualitative parameters that cannot be incorporated in a cost-effectiveness model may play decisive roles in the final judgment. For example, the decision to implement meningitis $C$ vaccine in The Netherlands may have partly been based on the judgment that it would reduce public anxiety on meningococcal disease (in addition to actually reducing the disease itself) as a clear and favorable cost-effectiveness analysis was not available. Furthermore, an acellular pertussis vaccine was introduced in The Netherlands in 2005 because of its better ratio of efficacy versus adverse events in comparison with whole cell vaccine, therewith trying to optimize confidence in the NIP. These examples illustrate that economic evaluations are not the only decisive factors in considering adjustments of the NIP.

Cost-utility analysis is the economic technique that expresses the utility of health care provisions in quality-adjusted life years or QALYs. Therewith, it becomes possible to compare the health burdens associated with different diseases, or health gains provided by different measures. Though introduced more than 25 years ago, a lively debate is ongoing on its usefulness, methodologies, and impact. This debate is nurtured by the assumptions and choices that are inherent to cost-effectiveness analysis. Uncertainty prevails in these analyses, including on the definition of the output of health gain, on its expression in QALYs, on the 
choice of the measurements that should provide comparable information across different diseases, on the choice of the procedure to value health, on the relative importance of the judgments of patients and the general public, on the quantification of various types of costs, and on the question as to whether and how future health gains should be discounted [8] and [10]. In addition, most of these analyses do not use infectious disease-specific dynamic models and neglect herd immunity. Vaccines may also afford real and perceived protection that cannot be accounted for in traditional cost-effectiveness analyses. Hence, there is no standard methodology for these analyses. For example, small health effects in many people may result in similar QALYs gained by an intervention as reduction of a very small number of deaths. Many people think that preventing severe or life-saving disease (even in a few individuals) merits highest priority, although often no consensus might be reached when specific choices have to be made [8]. There is also a wide belief that maximization of QALYs may conflict with considerations of fairness [11]. Other ethical issues of cost-effectiveness calculations are the question whether an additional year of life has the same value or health status regardless of the age of the person who receives it, the question whether reduction in quality of life by predisposing conditions or disabilities should be taken into account, the question whether a health gain is of greater value when it occurs sooner rather than later in life, and the question whether indirect benefits (lost wages, economic costs to employers, extra need child for care) should be included in the considerations. Cost-effectiveness analyses therefore cannot provide the final judgments required for deciding whether a particular investment to achieve a health effect is appropriate, fair, and just, or not.

\subsection{Unforeseen risks}

Although we foster that any decision is based on evidence as much as possible, some consequences of introducing a new vaccine or changing a schedule may not be foreseeable or predictable. A guiding principle is therefore that any decision should not be harmful ("first do not harm"). We would like to emphasize that the decision to introduce a vaccine in the NIP should be taken as seriously, both scientifically and ethically, as the decision to withhold (or even remove) a vaccine from the NIP. At first sight the principle of "first do not harm" might lead to passivity, but in that case one is also responsible for vaccine-preventable disease and mortality. Likewise, removing a public health service can be very difficult politically, unless there are clear benefits of safety or absence of benefit.

\subsection{Different goals of vaccination}

The reasons for including a vaccine in the NIP may vary. For example, polio vaccination nowadays is mainly done to eradicate the virus. If a RSV vaccine would become available, its main purpose is to diminish the consequences of a first infection in infected individuals. Polio vaccination thus has a greater public health impact than RSV vaccination (despite huge medical consumption associated with RSV hospitalization). Such individual interest in RSV vaccination may however be no reason to withhold it. It is important to stress that there is usually no conflict of interest between general and individual interests in vaccination. Exceptions may be when a very expensive vaccine (HIV) is being paid to benefit a few rather than purchasing a cheap vaccine (varicella) for the population at large, or refusal of individuals to vaccination therewith increasing the transmission of a pathogen. 


\subsection{Vaccination schedule}

A NIP is likely and perhaps necessarily a compromise, not only between epidemiological and economical factors, but also between practical considerations. For example, optimal protection may require different vaccination schedules for different vaccines. For practical and economic reasons a good "average" schedule for combined administration is chosen, that is not necessary optimal for each single vaccine component.

\subsection{Availability of vaccines}

A consideration for the choice of a particular vaccine may be its long-term availability and availability during outbreaks. Vaccine manufacturing capacity is clearly at stake [12]. Therefore, countries may strive towards national production facilities and expertise, which may also be of great benefit to counter-attack biological warfare and to produce commercially non-interesting vaccines for local markets. The argument is valid, but vaccines from local facilities should nonetheless meet the highest quality criteria, and potential conflicts of interests should be avoided by separating scientific advice on vaccination policy from vaccine production.

\subsection{Public perception and trust}

Despite some objections from religious and anti-vaccination groups, the confidence in NIPs is high given the high coverage rates. To ensure the public's trust in the program, information on adverse events and benefits should be collected and made public. The balance between presumed adverse and beneficial effects may however shift in time, for example, when the incidence of infection declines. Sporadic adverse events following vaccination may be considered acceptable if the benefits of vaccination are large.

The importance of trust in the vaccination program, and its vulnerability, has been shown recently in the United Kingdom, where vaccination rates with the combined measles mumps and rubella vaccine fell from more than $90 \%$ to less than $80 \%$ after unsubstantiated claims that the vaccine might cause autism (UK Department of Health, 2003). A rise in the incidence of measles and mumps followed. The suggestion was enough to cause this effect. In Sweden a decline in trust in the program caused a drop in vaccination coverage and an approximately 100 -fold rise in the incidence of pertussis [13].

Maintaining confidence in the NIP is thus top priority. Measuring and predicting the public's trust in the vaccination program and willingness to let one's children vaccinate, and determinants thereof, may require the development of new methods. Measuring the actual vaccination coverage is an important basic parameter, but perhaps not sufficient. Special attention is required for difficult-toreach isolated ethnic, religious, or social groups that oppose to vaccination, or that experience a language barrier. This is apparently a field of attention that is still in its infancy [14]. Often risk perception is seen against the background of public hope in combating devastating disease, and reluctance at being coerced into taking even small individual risk for the sake of society as a whole [15]. However, there is no society competing with individuals: both individuals and society as a whole carry (minimal) chances on unwanted side effects and gain (major) benefits by vaccination. (An exception would be hepatitis A vaccination of children, which mainly experience asymptomatic infection, to prevent disease transmission to adults.) 
Among the requirements for maintaining a high level of trust is a surveillance program monitoring the safety and effectiveness of the program, and good, consistent, open, evidence-based, referenced, and credible information on adverse events. Valid data about safety may counteract unsubstantiated safety concerns, such as the presumed MMR-autism link. Some countries, like the US, have implemented compensation for people who suffered serious adverse events that could be attributed to vaccination. In addition, more knowledge on the pathobiology of adverse events following vaccination is warranted.

Attention for practical considerations, such as assuring that the number of injections given at the same moment is not too high, may be necessary to assure a high coverage. An increasing number of injections might cause concern that a NIP becomes "overloaded", or that choice for a given vaccine excludes the use of others. To be clear, there is no evidence that the immune system may be antigenically overloaded by multiple immunizations [16]. Indeed the Dutch Health Council recently made a point of the number of injections that were considered acceptable (two at the same time was the perceived, but not scientifically proven, maximum number of acceptable injections [17]). Interestingly, in a questionnaire study, health care professionals were more concerned about multiple injections than mothers, and they were poor predictors of mothers' vaccine preference [18]. While most physicians had strong concerns about administering three or more injections at one visit, most parents in that study preferred this practice. Continued education and reassurance addressing concerns about multiple injections should therefore not only be directed to parents but also to physicians [19]. Nonetheless, there may be a competition between vaccines for entering the NIP. If true, it would mean that the success of a NIP would limit its further progress. Careful choice of vaccination schedules, the use of combination vaccines, careful education, and voluntary vaccination might combat this trend.

As an alternative to a comprehensive NIP, a government might offer a "small" NIP (for example, directed to diseases with the greatest public health impact and where herd immunity is most important to limit transmission), together with a voluntary package, assembled to the individual's needs and perceptions. The latter may be financed by public means, insurance companies, or by the individual(s' parents) self. However, such a development might lead to inequalities in the use of vaccine, thus setting the task for governments of assuring equal opportunities for vaccination and equal access to information. Additional advice on vaccination, tailored to the individuals' needs and risk factors, and the administration of vaccine might be given by specialized vaccination doctors or clinics.

Concern regarding trust in the program also applies when the NIP does not modernize rapidly enough according to the public's opinion. This may lead to considerable public and media pressure, lower vaccination coverage, and/or to the purchase and application of vaccines outside the framework of the NIP, as experiences in The Netherlands illustrate. Different and sometimes contrasting public opinions occur, ranging from "as few injections as possible" to "as much protection as possible" and "only the best for my child". Needless to say that it is no easy task to reconcile these contrasting views together, and with the policy maker's wish for a cost-effective program.

\subsection{Long-term effects}

Vaccines should not be introduced without assessment of possible long-term effects, and it should be acknowledged that we have still limited knowledge about, e.g., evolutionary consequences of vaccination. Unpredictable events, 
unwanted side effects or insufficient efficacy may follow. Often vaccination will not completely halt transmission and therefore exert an evolutionary force on the pathogen. Evolutionary consequences may include changes in antigenic composition (affecting the vaccine's efficacy), and changes in virulence and transmissibility, which are not easily predictable. Modeling predicts that vaccination against blood-stage malarial parasites could accelerate their rate of virulence evolution and make parasites more dangerous to non-immunized individuals [20]. Such a vaccine should therefore be combined with transmission blocking mechanisms to stop evolution. Immunizing chickens against a lowpathogenicity H5N2 influenza virus resulted in antigenic drift of the wild-type virus and reduction in the vaccine's efficacy. This probably contributed to the persistence of the wild-type virus [21]. In spite of worldwide vaccination since the 1950s, the incidence of pertussis is increasing again [22]. Several causes have been suggested for this resurgence, including improved diagnostics and surveillance, waning immunity, and the emergence of escape variants [23] and [24]. Vaccination may completely disrupt microbial transmission and thus stop microbial evolution. This appears superior to vaccination driving microbial evolution. However, disappearance of a pathogen might have evolutionary consequences on related micro-organisms by providing a new ecological niche. Other potential long-term consequences of vaccination are a shift in age distribution of infection, accompanied by changes in severity of disease or not. For example, varicella vaccination in children may reduce varicella-zoster virus circulation in the general population and thus cause waning population immunity, making the elderly (temporarily) more vulnerable for varicella and herpes zoster [25]. Finally, vaccination (or withdrawal of lipopolysaccharide from vaccines) might influence Th1/Th2 polarization of the immune system. Often the occurrence, direction, and magnitude of such long-term changes are speculative only, but they should be considered and estimated as accurately as possible. The fact that such long-term consequences might occur is in itself no reason to withhold the implementation of a vaccination. Indeed our capacity to take risks, be it as calculated and cautious as possible, is a prerequisite to make progress in matters like these. Surveillance should detect long-term consequences of vaccination, although cause and effect relations are difficult to establish.

\subsection{Universal or targeted vaccination}

To obtain a high level of herd immunity some countries have made their NIP obligatory, or have implemented some kind of psychological pressure. However, as the number of vaccines increases, it may not always be possible or required to apply a "one-size-fits-all" principle. This may require careful consideration of general versus individual interests. Especially when the availability of more vaccines (perhaps directed against less severe or less frequently occurring diseases) would lead to lower acceptance of the general program, then we might consider offering and advising such new vaccines without making them obligatory, or advising them with lower priority. Some parents may then choose for more vaccinations and a low risk on infectious disease, while others choose for fewer vaccinations and accept a low chance on serious disease. Needless to say (but difficult to understand and to communicate) that the distinction between severe and not severe is often artificial, as many infectious diseases have an unapparent or mild course in many individuals, and a severe to fatal course in only a few, the so-called "iceberg concept" of infectious diseases.

To illustrate the latter, varicella vaccination may reduce mild illness in many children and severe illness and death in rare cases, while a major economic benefit may be its capacity to be cost savings for parents who do not need time to take off from work to care for a sick child [6] and [8]. Hence, a mild course of 
infection in the majority of infected individuals appears no reason to withhold vaccination. Likewise, influenza in healthy children is usually not seen as a significant problem. However, influenza is not always benign and more important than previously thought. Bacterial complications, infections outside the respiratory tract (myositis, myocarditis, encephalitis), acute otitis media, and bronchiolitis may lead to a high rate in hospitalizations in children under 2 years [9]. Therefore influenza vaccination of infants aged 6-23 months, children with recurrent acute otitis media or respiratory tract infection, and healthy children attending day-care centers or elementary schools has been recommended. Before introduction of universal vaccination, Australia offered pneumococcal vaccination to large groups of high-risk children, i.e. all children in central Australia, all Aboriginal children, and all children at high risk due to pre-existing illness [26]. Similarly, in The Netherlands hepatitis B vaccination is offered, in contrast to the WHO's advice, to certain risk groups only.

If this trend continues, the NIPs may develop into vaccination programs that are tailored to age, life style, or other individual risk factors. If so, surveillance of the vaccination program should include monitoring of the vaccination coverage of the different risk groups, should examine the effectiveness of the program as a whole, and whether the program is sufficient to prevent spreading of the pathogen from the "risk groups" to the general population. A conflict of interest may thus arise between simplicity and uniformity of the NIP that may promote its acceptance [17], and the need or wish to tailor the program to individual needs. A wise compromise may reconcile these different premises.

Vaccination accommodated to age and life style may include vaccination against sexually transmitted diseases and against diseases transmitted during or early after gestation. These may be very cost-effective, and therefore one of the great opportunities for improving NIPs [8] and [27]. Candidate vaccines for "pubertal" or "adolescent" vaccination include those against HPV (for which successful trials have been reported [28] and [29]), Chlamydia trachomatis, herpes simplex virus type 2, hepatitis B virus, Neisseria gonorrhea, and group B streptococci, an impressive list that includes two anti-cancer vaccines. Evidently such an approach must be accepted by parents and their children, and this may require feasibility studies. Comparably, preventing infections in young children by vaccinating pregnant mothers (for example, against pertussis or RSV) may be a very effective concept, the application of which requires careful considerations of safety issues and liability concerns.

\subsection{Expensive vaccines}

Complex situations may occur when a vaccine is expensive (but cost-effective), or when it has an unfavorable cost-benefit ratio. Even when the vaccine-preventable burden of disease is high, calling for universal vaccination, high costs of a vaccine may prohibit its incorporation into a NIP. Wealthy and well-informed individuals may subsequently decide to buy such a vaccine for themselves or their children, resulting in inequalities that may be undesirable, but perhaps unavoidable.

\subsection{Surveillance of NIPs}

After a decision to change the program, its safety and effectiveness should be monitored carefully, both to evaluate current effectiveness (reflecting previous decisions), as well as to prepare for future changes. A thorough NIP surveillance should include the following elements: 
1. Clinical-epidemiological surveillance, measuring the effectiveness of vaccination in reducing the burden of disease, and in temporal trends thereof. It might be difficult however to judge whether temporal changes in the epidemiology of a disease are really vaccine-induced or merely coincidental.

2. Surveillance of vaccination coverage, which monitors the general acceptance of the program and identifies regions or groups with lower vaccination coverage that may endanger herd immunity. Monitoring trust in the NIP may be a useful addition to this activity.

3. Immune surveillance, monitoring markers of protection, and therewith the degree of protection in the general population or in subgroups thereof. Waning immunity and altered circulation of pathogens may, for example, make specific age groups more vulnerable to infection. For example, vaccine-induced reduction in measles virus circulation has reduced levels of maternal antibodies, demanding a solution to protect children earlier.

4. Surveillance of microbial population dynamics. As a consequence of natural or vaccine-driven evolution, pathogens may respond less to vaccination. This may necessitate the replacement of a vaccine strain by a better matching vaccine strain, a practice that is best known for influenza virus vaccines, but such surveillance is indicated for Hib, hepatitis B, meningococcal disease, mumps, and pertussis. While microbial evolution is mostly unpredictable, it shows that we have to take some uncertainty into account when deciding on the composition of the NIP.

5. Safety surveillance. Surveillance for adverse events following vaccination is necessary to define their frequency and nature, to optimize the program, to allow a comparison between benefits and adverse events of vaccination, and to help maintaining trust in the program. Comparing the benefits and risks of vaccination has, for example, led to the decision in many countries to replace the oral polio vaccine that in rare cases led to vaccine-associated paralytic polio, with the safer inactivated polio vaccine. Experiences with the mumps Urabe Am 9 vaccine strain [30] and an experimental rotavirus vaccine (RotaShield) [31] have shown that a good post marketing surveillance is necessary to detect rare serious adverse events that may necessitate the withdrawal of vaccines.

\subsection{Final remarks}

A decision on the introduction of a vaccine always brings some uncertainties with it, as some long-term consequences cannot be predicted, including antigenic changes, altered microbial interactions, and evolutionary consequences, but also future vaccine prizes and economic circumstances. However, reducing uncertainties and dealing with the remaining ones is at the core of medicine.

\section{References}

[1] H.-J. Schmitt, R. Booy, C. Weil-Oliver, P. van Damme, R. Cohen and H. Peltola, Child vaccination policies in Europe: a report from the summits of independent European vaccination experts, Lancet Infect Dis 3 (2003), pp. 103-108.

[2] F. Abbink, A.M. Buisman, G. Doornbos, J. Woldman, T.G. Kimman and M.A.E. Conyn-van Spaendonck, Poliovirus-specific memory immunity in seronegative elderly people does not protect against virus excretion, J Infect Dis 191 (2005), pp. 990-999. 
[3] Asch-Goodkin J. For tomorrow's children: vaccines on the horizonhttp://www.contpeds.com/be_core/content/journals/k/data/2004/0501/futureshot.html.

[4] M.N. Oxman, M.D. Levin and G.R. Johnson et al., A vaccine to prevent herpes zoster and postherpetic neuralgia in older adults, N Engl J Med 352 (2005), pp. 2271-2284.

[5] L.J. Erickson, P. De Wals and L. Farand, An analytical framework for immunization programs in Canada, Vaccine 23 (2005), pp. 2468-2474.

[6] Boot HJ, De Melker HE, Stolk EA, De Wit GA, Kimman TG. Assessing the introduction of universal varicella vaccination in The Netherlands, submitted for publication.

[7] B. Gruyer, N. Hughart and E. Holt et al., Immunization coverage and its relationship to preventive health care visits among inner-city children in Baltimore, Pediatrics 94 (1994), pp. 3-58.

[8] K.R. Stratton, J.S. Durch and R.S. Lawrence, Vaccines for the 21st century. Institute of Medicine, National Academy Press, Washington, DC (2000) US Department of Health; 2003.

[9] N. Principi and S. Esposito, Are we ready for universal influenza vaccination in pediatrics?, Lancet Infect Dis 4 (2004), pp. 75-83.

[10] J.N.D. de Neeling, Kostenutiliteitsanalyse; onzekerheden beperken toepasbaarheid, Ned Tijdschr Geneesk 148 (2004), pp. 1106-1110.

[11] A. Williams and R. Cookson, Equity in health. In: A.J. Culyer and J.P. Newhouse, Editors, Handbook of health economics vol. I, Elsevier, Amsterdam (2000), pp. 1863-1910.

[12] J.U.S. Cohen, Vaccine supply falls seriously short, Science 295 (2002), pp. 1998-2001.

[13] E.J. Gangaroza, A.M. Galazka, C.R. Wolfe, L.M. Philips, R.E. Gangarosa and E. Miller et al., Impact of anti-vaccine movements on pertussis control: the untold story, Lancet $\mathbf{3 5 1}$ (1998), p. 356

[14] Paulussen TGW, Lanting CI, Buijs GJ, et al. Ouders over het Rijksvaccinatieprogramma: tevredenheid en vaccinatiebereidheid van ouders van jonge kinderen in Nederland. TNO-rapport PG/JGD2000.033. Leiden, TNO Preventie en Gezondheid; 2000.

[15] M.A. Balinska, Vaccination in tomorrow's society, Lancet Infect Dis 3 (2003), pp. 443-447.

[16] A.L. Gregson and R. Edelman, Does antigenic overload exist? The role of multiple immunizations in infants, Immunol Allergy Clin North Am 4 (2003), pp. 649-664.

[17] Gezondheidsraad: Algemene vaccinatie tegen meningokokken C en pneumokokken.

Gezondheidsraad: Den Haag; 2001. publicatie nr 2001/27.

[18] B.A. Halperin, B.J. Eastwood and S.A. Halperin, Comparison of parental and health care professional preferences for the acellular or whole cell pertussis vaccine, Pediatr Infect Dis J 17 (1998), pp. 103-109.

[19] K.A. Woodin, L.E. Rodewald, S.G. Humiston, M.S. Carges, S.J. Schaffer and P.G. Szilagyi, Physician and parent opinions. Are children becoming pincushions from immunizations?, Arch Pediatr Adolesc Med 149 (1995), pp. 845-849.

[20] M.J. Mackinnon and A.F. Read, Immunity promotes virulence evolution in a malaria model, PLoS Biol 2 (2004), p. E230.

[21] C.W. Lee, D.A. Senne and D.L. Suarez, Effect of vaccine use in the evolution of Mexican lineage H5N2 avian influenza virus, J Virol 78 (2004), pp. 8372-8381.

[22] J.D. Cherry, Epidemiological, clinical, and laboratory aspects of pertussis in adults, Clin Infect Dis 28 (1999) (Suppl. 2), pp. S112-S117. 
[23] F.R. Mooi, H. van Oirschot, K. Heuvelman, H.G. van der Heide, W. Gaastra and R.J. Willems, Polymorphism in the Bordetella pertussis virulence factors P.69/pertactin and pertussis toxin in The Netherlands: temporal trends and evidence for vaccine-driven evolution, Infect Immun 66 (1998), pp. 670-675.

[24] F.R. Mooi, I.H. van Loo and A.J. King, Adaptation of Bordetella pertussis to vaccination: a cause for its reemergence?, Emerg Infect Dis 7 (2001), pp. 526-528.

[25] M. Brisson, W.J. Edmunds and N.J. Gay, Varicella vaccination: impact of vaccine efficacy on the epidemiology of VZV, J Med Virol 70 (2003) (Suppl. 1), pp. S31-S37.

[26] M. Howe, Pneumococcal vaccination programme begins in Australia, Lancet Infect Dis 1 (2001), p. 69

[27] M. Verweij, Ethische uitgangspunten voor het collectieve vaccinatieprogramma, Infectieziekten Bulletin 11 (2000), pp. 258-261.

[28] D.M. Harper, E.L. Franco, C. Wheeler, D.G. Ferris, D. Jenkins and A. Schuind et al., Efficacy of a bivalent L1 virus-like particle vaccine in prevention of infection with human papillomavirus types 16 and 18 in young women: a randomised controlled trial, Lancet 364 (2004), pp. 1757-1765.

[29] L.A. Koutsky, K.A. Ault, C.M. Wheeler, D.R. Brown, E. Barr and F.B. Alvarez et al., A controlled trial of a human papillomavirus type 16 vaccine, N Engl J Med 347 (2002), pp. 1645-1651.

[30] J.C. McDonald, D.L. Moore and P. Quennec, Clinical and epidemiologic features of mumps meningoencephalitis and possible vaccine-related disease, Pediatr Infect Dis J 11 (1989), pp. 751755.

[31] MMWR, Suspension of rotavirus vaccine after reports of intussusception-United States, Morb Mortal Wkly Rep 53 (2004), pp. 786-789. 